\title{
A process evaluation of the WHiTE Two trial comparing total hip arthroplasty with and without dual mobility component in the treatment of displaced intracapsular fractures of the proximal femur
}

\author{
CAN A TRIAL INVESTIGATING TOTAL HIP ARTHROPLASTY FOR HIP \\ FRACTURE BE DELIVERED IN THE NHS?
}

\section{Huxley, J. Achten, M. L. Costa, F. Griffiths, X. L. Griffin}

University Hospitals Coventry and Warwickshire NHS

Trust, Coventry, United Kingdom

n C. Huxley, PhD Psychology, MSc Health Psychology, BSc (Hons) Psychology, Senior Research Fellow,

- F. Griffiths, RCGP, PhD, Head of Division, University of Warwick, Warwick Medical School, Social Science and Systems in Health, Coventry, CV4 7AL, UK.

I. Achten, MSc Biological Health Sciences, PhD exercise biochemistry, Research Manager, - M. L. Costa, FRCS (Tr\&Orth), PhD Achilles tendinopathy and rupture, Professor of Orthopaedic and Trauma Surgery,

X. L. Griffin, FRCS (Tr\&Orth), PhD Medicine, Associate Professo of Trauma Surgery, University of Oxford, NDORMS, Kadoorie Centre, Level 3 John Radcliffe Hospital, Oxford, OX3 9DU, UK.

Correspondence should be sent to Mr X. L. Griffin; email: xavier. griffin@ndorms.ox.ac.uk

doi: 10.1302/2046-3758.510. BJR-2015-0008.R1

Bone Joint Res 2016;5:444-452. Received: 12 March 2016; Accepted: 11 May 2016

\section{Objectives}

The annual incidence of hip fracture is 620000 in the European Union. The cost of this clinical problem has been estimated at 1.75 million disability-adjusted life years lost, equating to $1.4 \%$ of the total healthcare burden in established market economies. Recent guidance from The National Institute for Health and Clinical Excellence (NICE) states that research into the clinical and cost effectiveness of total hip arthroplasty (THA) as a treatment for hip fracture is a priority. We asked the question: can a trial investigating THA for hip fracture currently be delivered in the NHS?

\section{Methods}

We performed a contemporaneous process evaluation that provides a context for the interpretation of the findings of WHiTE Two - a randomised study of THA for hip fracture. We developed a mixed methods approach to situate the trial centre within the context of wider United Kingdom clinical practice. We focused on fidelity, implementation, acceptability and feasibility of both the trial processes and interventions to stakeholder groups, such as healthcare providers and patients.

\section{Results}

We have shown that patients are willing to participate in this type of research and that surgeons value being part of a team that has a strong research ethos. However, surgical practice does not currently reflect NICE guidance. Current models of service delivery for hip fractures are unlikely to be able to provide timely total hip arthroplasty for suitable patients.

\section{Conclusions}

Further observational research should be conducted to define the population of interest before future interventional studies are performed.

Cite this article: Bone Joint Res 2016;5:444-452.

Keywords: Hip fracture, Total hip arthroplasty, Process assessment (health care)

\section{Article focus}

- The National Institute for Health and Clinical Excellence states that research into the clinical and cost effectiveness of total hip arthroplasty as a treatment for hip fracture is a research priority.
Can a suitable trial investigating total hip arthroplasty for hip fracture currently be delivered in the NHS?

- A mixed methods approach to situate a feasibility trial within the context of the United Kingdom's clinical practice. 


\section{Key messages}

- Patients are willing to participate in surgical research and that surgeons value being part of a team that has a strong research ethos.

- Current models of service delivery for hip fracture are unlikely to be able to provide timely total hip arthroplasty within the context of a trial.

\section{Strengths and limitations}

- Contemporaneous process evaluation to explain the context of the feasibility study: WHiTE Two.

- Multiple stakeholders engaged: patients, surgeons, allied health professionals, managers.

- Single centre, multi surgeon study.

\section{Introduction}

Hip fractures are one of the greatest challenges facing the medical community. In 1990, a global incidence of 1.31 million was reported and associated with 740000 deaths. ${ }^{1}$ The annual incidence of hip fracture is 620000 in the European Union. ${ }^{2}$ These figures constitute a heavy socioeconomic burden worldwide with the cost of this clinical problem estimated at 1.75 million disabilityadjusted life years lost, equating to $1.4 \%$ of the total healthcare burden in established market economies. ${ }^{1}$

Recent guidance from The National Institute for Health and Clinical Excellence (NICE) states that research into the clinical and cost effectiveness of total hip arthroplasty (THA) as a treatment for hip fracture is a priority. ${ }^{3}$ Despite several large randomised studies addressing this issue, the role of THA remains uncertain. ${ }^{4}$ Further funding to investigate the clinical effectiveness of THA in the United Kingdom is not expected to be made available until researchers can demonstrate why a trial is likely to be successful in changing clinical practice, provide insight into the mechanisms and processes responsible for any effect and address questions around the feasibility of the intervention in routine clinical practice. ${ }^{4}$

The Warwick Hip Trauma Evaluation Two study (WHiTE Two) (in press) was a single-centre, multi-surgeon, parallel, two-arm, standard-of-care controlled randomised pilot study investigating the dislocation risk of a dual-mobility acetabular component compared with a standard component in total hip arthroplasty. The study was limited to appropriately high functional demand patients with displaced intracapsular hip fractures who fulfilled the NICE criteria for THA. ${ }^{3}$ The study was embedded within the overarching Warwick Hip Trauma Evaluation Comprehensive Cohort Study. ${ }^{5}$ The full protocol for this trial ${ }^{6}$ and results (in press) have been reported elsewhere. This paper reports the outcome from a contemporaneous process evaluation that provides a context for the interpretation of the findings of the pilot study.

Process evaluations use empirical data to assess the delivery of programmes. ${ }^{7}$ Therefore, in contrast to the WHiTE Two randomised trial, which was an evaluation of outcomes, this process evaluation explored the nature of the trial protocol and whether it could be implemented as a larger multicentre study.

We sought to determine whether a multicentre trial of THA could be delivered in the NHS.

\section{Patients and Methods}

The methodology for this process evaluation was underpinned by the components identified by Linnan and Steckler: ${ }^{8}$ context, reach, implementation and recruitment. We developed a mixed methods approach to best enable us to place University Hospitals Coventry and Warwickshire NHS Trust in the context of wider United Kingdom clinical practice. The trial was registered (ISRCTN90544391) and included within the National Institute for Health Research Portfolio (122067). Ethical approval was granted on 1 May 2013 by the Coventry and Warwickshire National Research Ethics Service Committee (13/WM/0110).

Sampling. We had planned to use a purposeful sampling strategy to ensure a diverse mix of patients and carers with respect to the following factors: age, gender and pre-injury EuroQol five dimensions (EQ-5D) utility score. However, since the size of the sample within the feasibility study was small we approached all available participants. In order to ensure that a broad range of opinion was captured from the available surgeons, we surveyed all providers of the intervention within University Hospitals Coventry and Warwickshire NHS Trust.

Context. There is considerable variability in practice concerning THA for fracture. ${ }^{9}$ This may reflect either variation in clinical practice or differences in population demographics across the United Kingdom. We combined data from Hospital Episode Statistics and the National Hip Fracture Database (NHFD) to determine how this variability could be explained nationally. The methodology and findings are reported elsewhere. ${ }^{10}$ We explored the specific local context through surgeon interviews in order to describe best the setting of the feasibility study. We recorded important aspects of the patients' journey through the trial and the local systems in place for service delivery.

Reach. The degree to which the trial sampled from the total eligible population was assessed through careful maintenance of a screening log. At daily trauma meetings at University Hospitals Coventry and Warwickshire NHS Trust, a research associate assessed for eligibility all patients who had sustained a fracture of interest. Reasons for exclusion were documented. Reasons for all subsequent exclusions or protocol violations were also recorded, identifying any patient, care provider or system barriers to recruitment.

Participant experiences. Patients who had participated in the feasibility trial (full characteristics of patients are reported elsewhere; in press) were recruited to participate in an interview via telephone a minimum of one 
week after surgery. Contact was attempted with 12 people who had received an intervention and had been entered into the study. In total, ten people (nine female) agreed to participate in an interview; the remaining two people could not be contacted during this phase of the research, despite repeated attempts. At the time of interview, participants had undergone surgery between two and seven months earlier.

Participant interviews were semi-structured, up to 60 minutes long, and were conducted in patients' homes or via telephone. These interviews focused on participants' feelings about participation in the trial, specifically the post hoc consent procedure, the randomisation process, and the burden of follow-up. Additional questions concentrated on their hip trauma and recovery. The interview schedule was amended after four interviews to shift from focusing on events in chronological order to first focusing on the hip trauma, treatment and recovery, and then on the research process.

Delivery of the intervention. All trauma and orthopaedic consultant surgeons at University Hospitals Coventry and Warwickshire NHS Trust who participate in the delivery of the trauma service, were surveyed to explore their opinions about the trial protocol and provision of THA for fracture within the NHS. A total of 19 orthopaedic surgeons at the study site were opportunistically recruited during a Professional Development Session. Participants had a range of expertise, and several had more than one specialty, however, the majority $(n=8)$ had a specialist interest in hip/lower limb surgery. The majority, but not all, worked on trauma rotas and six had entered patients into the trial. In order to protect the anonymity of these participants, gender and exact specialty are not reported.

Surgeon interviews were brief (up to 15 minutes long), semi-structured, and were conducted in staff offices, meeting rooms and via telephone. The interview schedule focused on preferences for the standard component or the dual-mobility acetabular component being tested in the trial, how feasible it was to enrol patients in a trial in a semi-emergency situation, and whether there were any concerns about the post hoc style of consent.

Members of the British Orthopaedic Trauma Society were surveyed for their opinions regarding delivery of THA for hip fracture. A recruitment email was sent to the mailing list containing a brief description of the study and link to the survey webpage. A reminder email was sent after two weeks, and the survey was open for four weeks in total. A total of 38 people (24\% response rate) responded to the recruitment invitation. Respondents were predominantly surgeons who perform THA for any indication on a regular basis, although fewer regularly perform THA for hip fracture.

The nine-item survey compromised a mixture of closed $(n=5)$ and open $(n=4)$ questions. Closed questions focused on how often respondents performed THA, how confident they felt performing THA, and how willing they were to randomise patients to THA for hip fracture trials. Response options were multiple choice or 1 to 10 on the Likert scale, ${ }^{11}$ where 1 was unwilling or unconfident, and 10 was very willing or very confident. Open questions concentrated on why respondents felt confident (or not) to perform THA, and what concerns respondents had about randomising patients to THA trials and to trials conducted in semi-emergency situations. The front page of the survey explained why the survey was being conducted, what would happen to respondents' data, and how long the survey would take to complete. Participants were also informed that by completing the survey they were providing consent for their anonymous data to be analysed and published.

All qualitative data (interview transcripts and responses to open-ended survey questions) were analysed separately using inductive thematic analysis. ${ }^{12}$ The aim of this analysis is to identify key issues and patterns within the dataset. Thematic analysis involves a process of immersion in the data, initial coding and subsequent sorting of codes into meaningful themes. As the focus of the research was acceptability of the research processes, only data related to this topic were analysed. Data from closed survey questions were analysed using descriptive analysis only in order to inform the qualitative findings. The analyses from both types of survey question are integrated in the results. As the different components of the research focus on different issues, results from all three phases of the work are presented separately. When using direct participant quotes, we signal our editing (such as removal of stuttering) with a bracketed gap.

\section{Results}

Local context. University Hospitals Coventry and Warwickshire NHS Trust is a Major Trauma Centre serving a population of approximately five million patients. The Department of Trauma and Orthopaedic Surgery has 28 consultants and a further 12 Specialist Trainee and Staff Grade surgeons. Amongst this group, the orthopaedic trauma service is largely delivered by 20 surgeons.

In $2014,26 \%$ of patients who were potential candidates for THA $^{3}$ received a THA. ${ }^{9}$ This proportion lies approximately in the middle of the national distribution. ${ }^{9}$ Reach. A summary of the flow of participants through the study is shown in Figure 1. In total, 26 patients admitted with a displaced fracture of the intracapsular hip were assessed to be eligible for inclusion in the trial. In all, 21 participants were randomised, of whom one was withdrawn from follow-up. A total of two participants were lost to follow-up, so that the proportion of participants available for follow-up at one year was $90 \%$.

Recruitment. While several participants had fairly clear recall of when and what they were told about the study, nearly all participants expressed some lack of recall, either about the study as a whole, or about specific details of the study. Some participants did not recall participating 


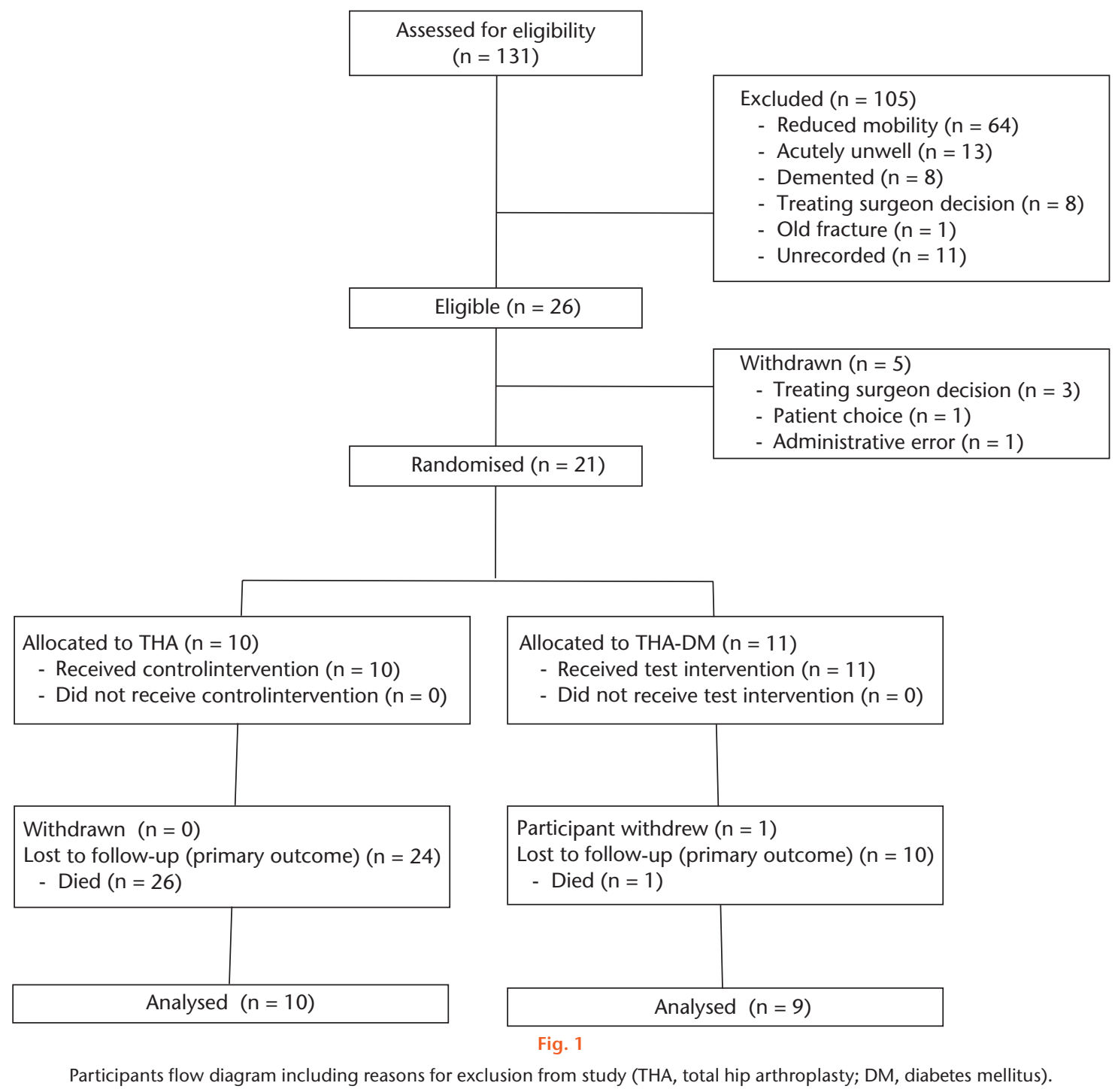

at all until receiving the information sent to them about the interviews:

“They didn't tell a lot, you know, I just signed a form and I just waited to hear from you. So I mean when I got that [information about the interview component of the research], you know, it told me a bit more" (P010).

Several of these people emphasised that their lack of recall was due to their physical and emotional stress in hospital. Participants described how being "in so much pain" (P004) and "frightened to death" (P009) caused their lack of recall of this time. Because of the physical and mental trauma these people were experiencing, participating in a research project was not at the forefront of their minds either before ("I think my mind was just on my op" (P009) or after the operation ("it didn't worry me so long as I got back on my feet" (P006).

Surgeons tended to distance themselves from identifying specific barriers to recruitment for this trial and

barriers within their department. They talked instead about 'possible' barriers and their experiences at other organisations. Interviewees tended to focus on surgeonspecific barriers, but patient preferences for specific treatments and practical barriers were also mentioned. Practical barriers to recruitment focused on the need to have available the correct staff (to perform both randomisation and the surgery) and the correct equipment. Availability of the correct staff was cited as a potential problem at weekends and out of hours, but also more generally: "getting the right surgeon in the right place to deliver the intervention is actually [...] an issue" (S027).

Surgeon expertise at performing THA was cited as a common barrier for recruitment. It was both witnessed and speculated that non-hip specialists perform hemiarthroplasty instead, preventing patient recruitment to the study: "there's been situations where um patients are suitable for the trial and [...] then they've not gone into 
the trial because [the surgeon] might not want to do a hip replacement so they've ended up getting a different hip hemiarthroplasty or something" (S020).

In total, two surgeons commented that they felt uncomfortable telling patients that the research was being conducted because they did not know the best treatment for hip fracture. This caused discomfort either because they felt incompetent in telling patients that they did not know which treatment was best or because they felt that they did know which the best treatment was (based on available evidence and their experience) and so they felt that they were not being truthful with their patients: "I know what I would normally do with them and so I almost feel like I' $m$ fibbing to the patient slightly by telling [them] that we don't know the answer because maybe inside I do, but I try and not be biased in the way I present the evidence to them. But it is hard, I feel like I' $m$ fibbing to them sometimes" (S011).

Lack of awareness of the trial or knowledge of the inclusion criteria were also mentioned as possible barriers to recruitment. With a variety of different research projects running concurrently it can be difficult to keep everything in mind: "With the best will in the world it's sometimes not possible to be on top of all the trials that are going on" (S013). There was a suggestion that staff enthusiasm for research could be a potential problem at other organisations, because recruitment was seen to be "onerous" (S022); this was prefaced by the statement that this is not a problem at their centre.

Consent. Generally there was no dissatisfaction amongst the participants with the consent process. Several people confirmed that they could say 'no' when they were asked and felt comfortable that they had a choice. One person commented that it would not have been possible to ask her to consent prior to her surgery due to her emotional state:

"I don't think anybody could ask me whether I would be interested [in participating prior to the operation]. I mean I was in so much pain all the while and I was having so many people coming in..." (P004). Only one person expressed discontent about the consent procedure. This person was concerned that she wanted to make a choice whether to participate or not: “I don't think l'd be very pleased actually if I found out afterwards [...] if I had a choice l'd rather be told that they were doing this study and would you like to be part of it, rather than you are part... well, it's been randomly decided which hip you will have, you know" (P012).

This person was confident that the research was discussed with her prior to her surgery, and that she physically consented to participate afterwards.

An overwhelming view amongst the surgeons was that if the research processes had been approved by the National Research Ethics Service then the research processes were acceptable and did not require further discussion: "If it's approved by all the boards and the ethical board I haven't got a problem with it at all" (S005).
Another strong view was that the consent procedure was acceptable because patients would experience very little difference between the two arms, and both treatment arms used "tried and tested" (S022) components. As the patients themselves would undergo the same process from surgery to rehabilitation, there was less imperative to gain consent for randomisation prior to surgery:

"it depends whether or not um it will make a difference to them [...] if it's a small difference in the type of component and not a different operation [...] then yes they need to have an understanding of what the trial means, but it's less important I suppose than if they have to choose between two different treatments." (S013)

Several surgeons also queried whether participants could really give informed consent to the research in an emergency situation. Patients are in pain from their hip fracture, under the influence of pain relief medications and psychologically distressed, and are therefore not able to give their full attention to information about the research: "in the circumstances where you're operating on the next available emergency slot, I think for most of those cases the patients are, you know, bewildered, disorientated, and have also just been filled up with massive slugs of intravenous morphine, and then we give the patient information sheet and it just makes no sense to me. So I'm completely comfortable with the process in that emergency situation" (S027).

Patients were seen to have other priorities than research when in these situations, being concerned only that "their pain has gone away" (S002). Post hoc consent was described as an "ingenious" method for gaining consent for research involving trauma patients "where often you can't get consent pre-operatively" (S010).

While many people were enthusiastic about post hoc consent, caution was expressed against the widespread use of this approach. This approach is appropriate for emergency rather than elective surgery, particularly involving older populations who might be more confused, but, as one person stated, they are "not quite sure how to defend it in a younger, more alert group of patients" (S003). Use of this approach requires "a very clear reason why you couldn't give them due, you know, consent before hand" (S011).

A small number of people did express concerns about the post hoc consent procedure used in the randomised control trial (RCT). These tended to focus on discomfort at the contrast between this method and accepted ethical consent procedures. These people were "wary", "anxious" and "uncomfortable" about gaining patient consent for the research after being randomised into the study because this method of consent is unusual and very different from other practices:

"the fact that they haven't been, really been consented, you've just done it and then told them you've done it, which I find is at loggerheads with everything else that you do" (S018). It was stressed that there are not many 
trials without opportunity to talk to the patient before surgery (it's not that they're going to theatre straight away" S025) and in cases where this was possible, consent must be sought. Professionals who expressed concern often became more accepting of the current approach as the potential physical and mental state of the patient was discussed: "Is it the right thing to do? Well um probably I think those patients could be consented beforehand. [...] I guess sometimes in an open fracture situation, however, there will be lot of patients who may have polytrauma and awful injuries, um, and actually that [pre-operative consent] may not be appropriate at that stage." (S016).

Those who had reservations about this consent procedure tended to defer to the decisions of ethics committees, despite feeling personally uncomfortable: "as long as the ethical committee people and everyone else is happy with it I'm okay going along with it, but it still makes me a bit uncomfortable" (S018).

Randomisation. There was a general lack of awareness and understanding among participants about the randomisation process, and exactly what this meant for their treatment. Again, recall about the randomisation process was patchy. Most people were not aware (or could not recall) exactly how randomisation took place, and many felt that the first time this was explained to them was during the interview. Some participants expressed concern about the randomisation process, fearing that they had been allocated a component that was not as good as the other, or was not tested as rigorously. Some feared that they were "guinea pigs" (P004) with new hip replacements being tested 'on' them. One person in particular stressed that she expected to be told if she was involved in trialling a new or untested component. This person reassured herself that the lack of information provided about randomisation and the different components suggests that she has had a "tried and tested" hip replacement:

"I would expect them if it was that situation to say that 'yours might be experimental, but we think this is going to be good but we haven't tried it', I would have wanted to know that. Nobody said that to me so I'm assuming what I had was a tried and tested one. I think if you're going to have anything that's new you need that explaining to you, and that wasn't explained to me, so I don't think I did" (P008).

Other people were not concerned about the randomisation process. More important for them, was that the surgery they had undergone was successful and that everything was "working alright" (P007). There were, however, some misunderstandings about the randomisation process even after this had been explained again in the interview. There was still a feeling that surgeons chose the type of hip replacement used for each patient because they "know what's best for me. [...] you know with my history" (P001). These feelings were linked to the participants' trust in their surgeon to provide the best possible individual treatment.
Burden of follow-up. There was no negativity about the burden of follow-up for participants, and their comments indicated that most participants felt that they had not needed to dedicate significant amounts of time to the study. The majority of participants recalled receiving follow-up phone calls but this was not seen as burdensome, particularly as it did not involve travelling back to hospital or being physically tested in any way.

Other people could not recall participating in any followup activities, and some suggested that the interview was the only follow-up activity they had engaged in. This could be due to the relatively minimal time commitment required for the RCT follow-up. One person exemplifies this; first she states that she has not done any follow-up activities, and then recalls the follow-up phone calls. She dismisses these as not being in-depth, unlike the interview, and in doing so implies that trial follow-up is not a burden:

"Nothing I don't think. You're the first person that's... they phone sometimes and say 'how are you?' but I mean this is the first time we've gone into great depths. Yes 'we're from the research department, you know, how are you?' but they've never asked all these sorts of questions before." (P004)

Interventions. There was much discussion about what material the components were made of, and this was linked to speculation about which component they had been allocated. Only two people were not curious about which component they received. More people were curious, however, and several asked if they had received "the newer one" (P010) or "one of the older ones" (P008). Many expressed interest in finding out to satisfy their own curiosity.

Opinion was divided when asked which component they would choose if offered a choice. The majority of people said they would require more information before deciding. Based on their recall of the study information, some said they would choose the standard component: "if they told me that the standard hip joint was a proven one, obviously l'd choose that. But if they said to me that the new hip job they're going to try out they didn't have any sort of concrete evidence that it was going to be alright, I would have refused it." (P007).

Others, however, would prefer the newer component because of the assumption that "it must be better if it's newer" (P009). Only one person was able to recall details about the dual-mobility component being less prone to dislocation, and this was referred to in the discussion as to which component they would choose: "if the new one is less likely to dislocate or it's been structured so that it's less likely to then yes l'd much rather go with that" (P012). Several people expressed no opinion as to which component they would receive, suggesting that they wouldn't mind as long as surgery was effective, and that their surgeon did 'their best'.

Surgeons' preferences for, and familiarity with, one of the treatment arms was reported as an issue. The 
Table I. Frequency of responses to questions about confidence performing THA and willingness to randomise patients to receive different components in THA

\begin{tabular}{|c|c|c|}
\hline \multirow[t]{2}{*}{ Response ranges } & \multirow{2}{*}{$\begin{array}{l}\text { How confident do you feel } \\
\text { performing a THA for a hip fracture? } \\
\%(n)\end{array}$} & \multirow{2}{*}{$\begin{array}{l}\text { How willing would you be to randomise patients to } \\
\text { receive different components in THA for hip fracture? } \\
\%(n)\end{array}$} \\
\hline & & \\
\hline Not confident / willing ( 1 to 3 on scale) & $24(9)$ & $16(6)$ \\
\hline Somewhat confident / willing ( 4 to 7 on scale) & $24(9)$ & $34(13)$ \\
\hline Very confident / willing ( 8 to 10 on scale) & $52(20)$ & $47(18)$ \\
\hline Missing & $0(0)$ & $3(1)$ \\
\hline
\end{tabular}

THA, total hip arthroplasty

standard component was preferred by six surgeons, dual mobility by two, and the remaining interviewees expressed no preference. The standard component was preferred because it was familiar: "simpler, cheaper, and probably more reliable in my hands" (S027), whereas preferences for the dual mobility were based on the advantages it offers: "I don't think it particularly has any downsides as compared to the standard hip replacement. So I can see advantages to it, but not many disadvantages to it, apart from the additional cost" (S014).

Treatment preferences were strongly linked with familiarity. More people $(n=13)$ were unfamiliar with the dual mobility component and uncemented components than familiar, as these were not used within everyday practice: "for elective work, you know, I use standard prostheses, I don't, you know, have much occasion to use a dual mobility" (S001). This lack of familiarity was linked to a lack of confidence and competence using dual mobility and uncemented components. As expected, lack of familiarity was also based on specialty; non-hip specialists on the trauma rota would refer emergency hip surgery to hip specialists: "the vast majority of the time it can wait, doesn't need to be done as an emergency, so the morning after the on-call I hand it over to one of the hip surgeons" (S030). Only four interviewees expressed familiarity, confidence and competence using non-standard components for THA.

National context - confidence to perform THA. Members of the United Kingdom Orthopaedic Trauma Society were asked to indicate their confidence in performing THA for hip fracture on a scale of 1 to 10 ( 1 being unconfident and 10 very confident). A range of responses were reported, but the majority reported feeling 'very confident' (Table I).

Surgeon confidence in performing THA for hip fracture was predominantly shaped by their experience, particularly by the frequency with which they perform the surgery. Those who had highest levels of confidence reported performing the surgery regularly, had extensive experience of the surgery ("Been doing it for years"), were trained in the surgery, or were hip specialists. This contrasts with people who were not hip specialists or did not often perform the surgery ("Not done total hip replacements for 12 years - rota set up for arthroplasty surgeons to do total hip replacements for fracture") and who reported low levels of confidence. Only three people reported that concerns about "dislocation rate" or "higher complication rate" influenced their feeling of confidence.

National context - willingness to randomise patients in a THA trial. Most respondents reported being willing to randomise selected hip fracture patients into a trial involving THA: $84 \%(n=32)$ said that they would (missing data from $n=1$ ). Reponses to the open-ended questions provided a contrast, however, as several people reported reservations about randomisation relating to their patients. These concerns focused predominantly on patient inclusion criteria and available operating time. For example, one person was concerned that the volume of their patients was "not large enough" for successful randomisation. A small number of people reported concerns related to surgeon treatment preferences "within an overall group of patients who might be eligible for entry into this trial one would still have views about how individuals ought to be treated", or to structural factors "In our institution those who have total hip replacement get this done by the dedicated arthroplasty team. Those who get a hemi get it by an ST3 (Higher surgical specialist trainee at year 3) on a trauma list. The selection bias makes this trial unfeasible".

A number of reservations were expressed about randomisation based on the various possible treatment arms. Several people said that concern "Depends on the arms of the trial", while others expressed preferences for specific treatments "Would not be prepared to compromise on using posterior approach. Would not be prepared to use anterolateral. Would not be prepared to use an uncemented stem". Finally, a small number of more logistical reservations were expressed, such as scheduling and availability of expertise. Time was also explicitly mentioned; "Time in theatre" and "operating time on trauma lists".

When asked how willing respondents would be to randomise patients to receive different components in THA for hip fracture, a range of answers were reported. Nearly half of respondents were very willing to randomise patients, but there was also a lack of willingness among $18 \%$ of participants (Table I). Again, many people reported having no concerns about randomising patients to such trials, however, some described concerns about 
gaining consent, particularly the "Difficulty in consenting" and "time for getting a valid consent". Allowing sufficient "time for patients to think about it" was linked to delays in performing the surgery and increased time for patients in hospital.

\section{Discussion}

We have reported, to our knowledge, the first contemporaneous process evaluation alongside the report of a randomised trial in orthopaedic surgery. This evaluation has helped to contextualise the setting of the trial within the framework of United Kingdom trauma service provision, explore possible barriers to recruitment and the burden of follow-up for participants.

Our findings are strongly positive for conducting clinical research with patients with a hip fracture. Historically, research in this setting with this group of patients has been limited due to perceived issues surrounding consent, recruitment, randomisation and follow-up. We have shown that patients are willing to participate in this research and that surgeons value being part of a team that has a strong research ethic.

There are specific issues surrounding delivery of a future trial investigating THA for hip fracture, and the majority of these concerns are specific to the surgeon. Appropriate concerns are held by surgeons surrounding safeguarding their patients' best interests, including appropriate consent processes and their ability to provide high-quality treatment. Increasing surgical subspecialisation and the availability of surgeon-level data from the National Joint Registry ${ }^{13}$ inevitably mean that some surgeons do not feel equipped to offer THA to their patients.

Our study has highlighted that surgeons who do not offer THA as part of their elective practice may be unfamiliar with more specialist arthroplasty options that may offer potential advantages in this group of patients. Each of the surgeons who entered patients into the study were confident implanting both systems, removing concerns about learning curves. However, our wider discourse with the general on-call group exposes the limitations that the surgeon's mix of skills might impose on the design of a trial. Local systems that are put in place can be expected to vary between centres and designing a protocol to accommodate this variation may be challenging.

The starkest finding from this study is the lack of reach of the interventions - that is the degree to which THA is actually offered to those patients who are deemed 'eligible'. Only $20 \%$ of 'eligible' patients, as defined by NICE guidance, ${ }^{3}$ were included in this study. Despite multidisciplinary assessments made at admission as part of the NHFD dataset, ${ }^{14}$ surgeons felt that many patients were insufficiently mobile or well enough to be offered a THA. This finding is echoed in the NHFD report, where very substantial variation in delivery of THA can be seen, ranging from none to almost $60 \%$ of eligible patients receiving THA. ${ }^{9}$ The conclusion from this must be that surgical practice does not currently reflect NICE guidance. As part of our process evaluation we have explored possible patterns of clinical decision making that might explain which patients are offered THA. ${ }^{15}$

Prior to embarking on an expensive multicentre trial investigating the clinical effectiveness of dual-mobility acetabular components or large head femoral components, ${ }^{3}$ we should first develop systems to provide THA in a timely fashion to appropriate patients. It is clear from our study that not all surgeons participating in on-call rotas are confident in providing such a service so alternative local solutions should be sought. Options might include access to elective hip lists on an urgent basis with reserved space for patients with a fracture or the provision of multiple trauma lists each day with an appropriate mix of surgeon subspecialty interest e.g., upper and lower limb biases. It is interesting to note, however, that THA is an index procedure for training in United Kingdom and Ireland; trainees are required to perform 40 cases, to be competent to perform cases unsupervised and to deal with complications; yet we have a cohort of consultant surgeons - many of whom will have trained under these or similar requirements - who are not able to perform this procedure.

Secondly, we should try to better define the characteristics of the population which we believe as trauma surgeons benefits from THA. Such a research question is perhaps not best addressed through a randomised trial but rather using observational study designs. The orthopaedic trauma community and others have invested considerable effort into compiling cohorts with patientreported outcomes, ${ }^{16}$ as well as the largest database for hip fracture in the world. ${ }^{14}$ Perhaps it is time to revisit our national guidance based upon findings from these resources.

\section{References}

1. Johnell 0, Kanis JA. An estimate of the worldwide prevalence, mortality and disability associated with hip fracture. Osteoporos Int 2004;15:897-902.

2. Svedbom A, Hernlund $\mathbf{E}$, Ivergård $\mathbf{M}$, et al. Osteoporosis in the European Union: a compendium of country-specific reports. Arch Osteoporos 2013;8:137.

3. No authors listed. The Management of Hip Fracture in Adults. London, National Clinical Guideline Centre: 2011. https://www.nice.org.uk/guidance/cg124/documents/ hip-fracture-full-guideline2 (date last accessed 08 August 2016).

4. Carroll C, Stevenson M, Scope A, Evans P, Buckley S. Hemiarthroplasty and total hip arthroplasty for treating primary intracapsular fracture of the hip: a systematic review and cost-effectiveness analysis. Health Technol Assess 2011;15:1-74.

5. Griffin XL, Achten J, Parsons N, et al. The Warwick Hip Trauma Evaluation - an abridged protocol for the WHiTE Study: A multiple embedded randomised controlled trial cohort study. Bone Joint Res 2012;1:310-314.

6. Griffin XL, McArthur J, Achten J, Parsons N, Costa ML. The Warwick Hip Trauma Evaluation Two -an abridged protocol for the WHiTE Two Study: an embedded randomised trial comparing the Dual-Mobility withpolyethylene cups in hip arthroplasty for fracture. Bone Joint Res 2013;2:210-213

7. Moore GF, Audrey S, Barker M, et al. Process evaluation of complex interventions: Medical Research Council guidance. BMJ 2015;350:h1258.

8. Steckler A, Linnan L, eds. Process Evaluation for Public Health Interventions and Research. First ed. San Francisco: John Wiley \& Sons, Inc., 2002. 
9. Boulton C, Burgon V, Cromwell D, Johansen A, Stanley R, Tsang C, et al. National Hip Fracture Database National Report 2014. http://www.nhfd. co.uk/2014report (date last accessed 12 May 2016).

10. Chaudhry H. Total hip arthroplasty after hip fracture. BMJ 2016;353:i2217.

11. Likert R. A technique for the measurement of attitudes. Archives of Psychology 1932;140:1-55.

12. Braun V, Clarke V. Using thematic analysis in Psychology. Qual Res Psychol 2006;3:77-101.

13. Perry DC, Parsons N, Costa ML. Surgeon level data: understanding the plot. Bone Joint J 2013;95-B:1156-1157.

14. No authors listed. The National Hip Fracture Database. 2015 Annual Report. http:// www.nhfd.co.uk (date last accessed 31 May 2016).

15. Perry DC, Griffin XL, Costa ML. Inequalities in use of total hip arthroplasty for hip fracture: population based study. BMJ 2016;353:i2021.

16. Griffin XL, Parsons N, Achten J, Fernandez M, Costa ML. Recovery of healthrelated quality of life in a United Kingdom hip fracture population. The Warwick Hip Trauma Evaluation-a prospective cohort study. Bone Joint J 2015;97-B:372-382.

\section{Funding Statement}

This was an investigator-initiated, industry funded trial. A research grant has been received from Orthodynamics by the University of Warwick which is related to this article.

- Grants have also been received from NIHR, ARUK and X-Bolt for research into Musculoskeletal Trauma which are not related to this article.

Author Contribution

C. Huxley: Interviews, Data collection and analysis

- J. Achten: Study inception, Manuscript preparation

- M. L. Costa: Study inception \& Manuscript preparation

F. Griffiths: Data analysis, Manuscript preparation

X. L. Griffin: Study inception, Manuscript preparation, Guarantor

ICMJE conflict of interest

None declared

(c) 2016 Griffin et al. This is an open-access article distributed under the terms of the Creative Commons Attributions licence (CC-BY-NC), which permits unrestricted use, distribution, and reproduction in any medium, but not for commercial gain, provided the original author and source are credited. 